\title{
LA-UR-13-23749
}

Approved for public release; distribution is unlimited.

Title:

Author(s):

Intended for:

Issued:

\section{Report of Campaign 3 Imaging Systems Planning Workshop}

Barnes, Cris W.

Report

2013-05-23

\section{- LosAlamos NATIONAL LABORATORY

\author{
EST. 1943
}

Disclaimer:

Los Alamos National Laboratory, an affirmative action/equal opportunity employer,is operated by the Los Alamos National

Security, LLC for the National NuclearSecurity Administration of the U.S. Department of Energy under contract DE-AC52-06NA25396. By approving this article, the publisher recognizes that the U.S. Government retains nonexclusive, royalty-free license to publish or reproduce the published form of this contribution, or to allow others to do so, for U.S. Government purposes.

Los Alamos National Laboratory requests that the publisher identify this article as work performed under the auspices of the

U.S. Departmentof Energy. Los Alamos National Laboratory strongly supports academic freedom and a researcher's right to publish; as an institution, however, the Laboratory does not endorse the viewpoint of a publication or guarantee its technical correctness. 


\section{Report of Campaign 3 Imaging Systems Planning Workshop March 25-26, 2013}

\section{Los Alamos National Laboratory, LANSCE National User Facility}

\section{Executive Summary}

Many mission applications for NNSA NA-10 use dynamic imaging. These include radiographic systems for hydros and focused experiments as well as smaller-scale science experiments, both present and future. Better understanding and reduced uncertainties would be possible with improved imaging systems. While each application has different specific requirements and issues where technical advances could improve the measurements, there are areas of common need where prioritized research and development could advance imaging systems for multiple applications.

A workshop was held March 25-26, 2013 at Los Alamos with about 44 invited participants from NNSA sites, industrial partners, universities, and key leaders in detector research from international research facilities. Introductory plenary talks focused on general challenges, needs, and requirements as well as opportunities and new directions in imaging systems. The workshop then had three parallel "breakout" sessions aligned along mission applications:

1. Ultrafast Applications, generally less than nanosecond time scales, such as found at high-energy-density physics facilities;

2. Fast Applications, generally nanosecond to microsecond time scales, such as found at light sources; and

3. Penetrating Radiography Applications, where single to several frames of imaging data are obtained on thick dynamic objects.

The participants identified a few Priority Research Directions for each breakout session, and after describing the direction determined which challenges and opportunities and what requirements each direction addressed, and what the advantages and impact of each would be. Summaries of those breakouts are within this report, including some cross-cutting directions or areas of investment. They can be combined independent of breakout application area (recognizing the requirements and challenges of the broader directions expand) into the following set:

1. Development of the "Universal Gated Detector," both a "backplane" as well as different optimized application specific "detectors." It would be a multiframe $(\sim 10)$ imager for optical and soft $\mathrm{x}$ rays.

a. And further development into low noise-floor $\left(<10 \mathrm{e}^{-}\right)$for photonstarved applications

b. And further development into phosphors that allow use of hard (2060-keV) x rays 
2. Interactions with materials scientists and industrial partners on controlling the properties of desired scintillators

3. More application specific needs:

a. Ultrafast few-picosecond imaging

b. Gated single frame imagers

c. Direct energy detection (of $\mathrm{MeV}$ photons) imagers

4. "Annual" C3-sponsored information workshop

5. Ensure the Infrastructure in place, both people (collaborations) and equipment (testing and characterization)

\section{Contents}

Report of Campaign 3 Imaging Systems Planning Workshop ............................................. 1

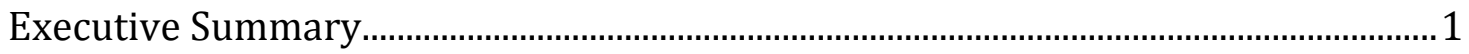

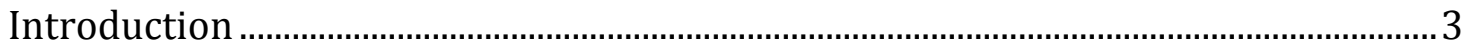

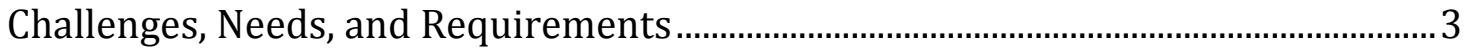

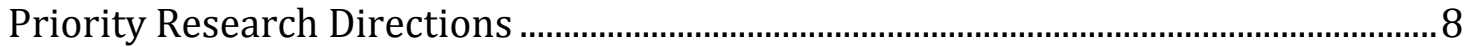

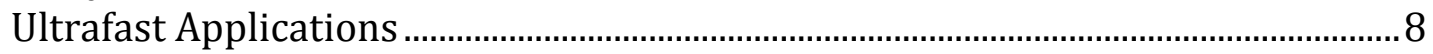

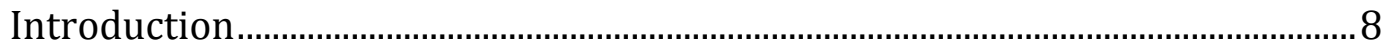

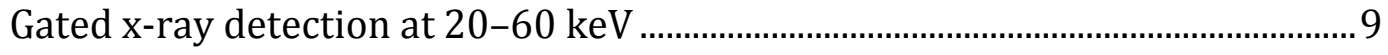

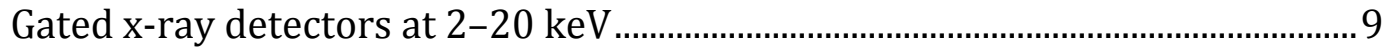

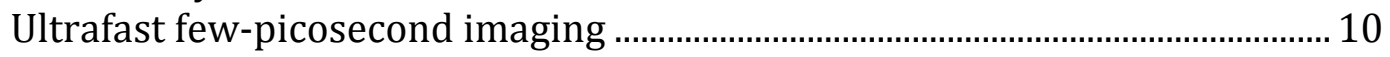

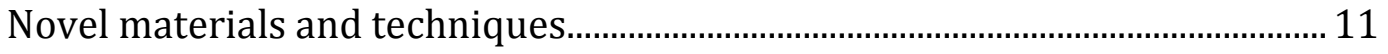

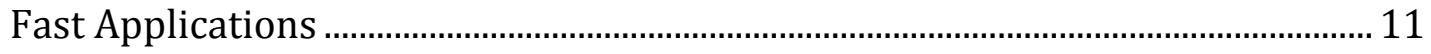

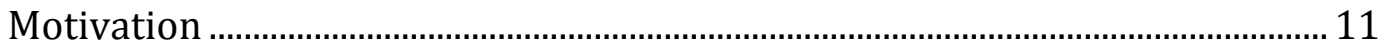

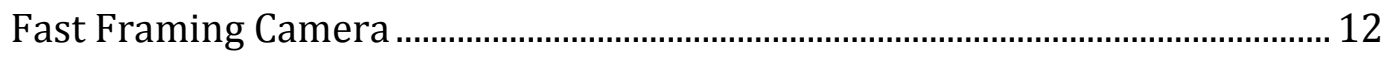

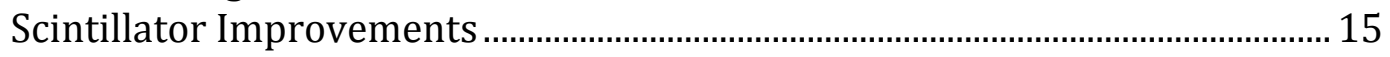

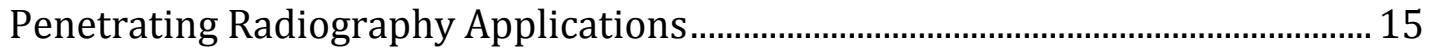

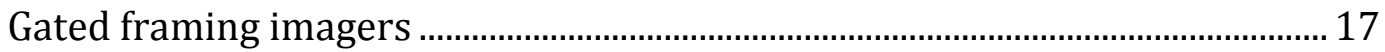

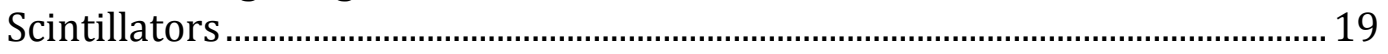

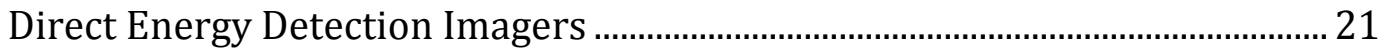

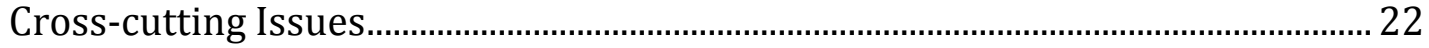

Annual C3-sponsored information workshop: ....................................................... 22

Ensure the Infrastructure in place, both people (collaborations) and

equipment (testing and characterization):............................................................... 22

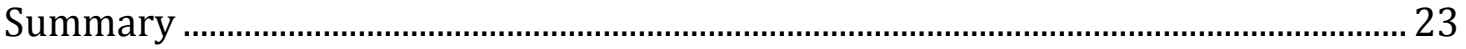

Final Agenda

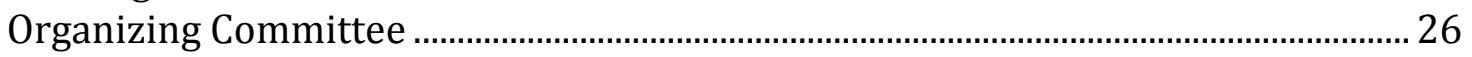

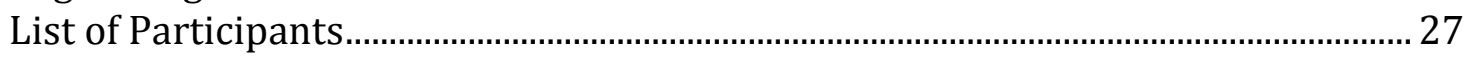




\section{Introduction}

Many mission applications for NNSA NA-10 use dynamic imaging. These include radiographic systems for hydros and focused experiments as well as smaller-scale science experiments, both present and future. Better understanding and reduced uncertainties would be possible with improved imaging systems. While each application has different specific requirements and issues where technical advances could improve the measurements, there are areas of common need where prioritized research and development could advance imaging systems for multiple applications. High impact could occur in the development of better detectors as well as in the conversion process from the hard-energy probe particles (photons, protons, or neutrons) to electronic signals. In particular, development of "higher-speed" (able to create more imaging frames of electronic information on faster time scales) and "higher-resolution" (many pixels in appropriate spatial format) systems that achieve "high-efficiency" (low noise with improved efficiency of converting hard energy incident particles into electrons) are possible cross-cut areas.

Campaign 3 sponsored an invitation-only meeting that brought together representatives from the NA-10 sites working on imaging system development, along with a few key people from industrial vendors and important detector research laboratories. ${ }^{1}$ About 45-50 invitees attended. This is the workshop report providing recommendations for NNSA investment in priority research directions in imaging systems that can cross-cut many mission applications.

A clear piece of advice arose from experts involved in previous development efforts: It is very important to ask the right questions and set the right metrics for the project. Then, with commitment and a sufficiently diverse and dedicated team, success can follow.

\section{Challenges, Needs, and Requirements}

This workshop confirmed the utility of community-based interaction and planning in developing and prioritizing research directions. Bringing application users together with device developers and key industrial partners allowed discovery of strong commonality of interest and new approaches that appeared to be broadly applicable.

There was agreement that "united we stand, divided we fall" or fail. It is possible to solve even major challenges with long-term, dedicated efforts. However, experience teaches us that the challenges outlined in this report require a long-term (decade) and expensive (cumulative few tens of million dollars) effort. Too often single-site development efforts that are sub-critical hit a problem and have insufficient resources to overcome that problem and subsequently fail. The cost of development of new technology may be greater than the benefit which will accrue to a single program trying to pay for the investment. Investments planned and executed

\footnotetext{
${ }^{1}$ See the web site http://lansce.lanl.gov/c3imaging , including the pages with links to the workshop materials.
} 
"complex-wide" (multi-sites) may be able to overcome barriers that single-site efforts cannot. Achieving consensus requirements, "good goals" set up front by multiple projects, may result in systems with broad applicability with greater interest for more such devices, allowing the development costs to be amortized across more projects. An example of such a major detector development effort is shown in Figure 1, the Adaptive Gain Integrating Pixel Detector (one of three such systems under development at EXFEL). How to implement some common planning and execution is a C3 program decision, one we recommend the Science Executives consider.

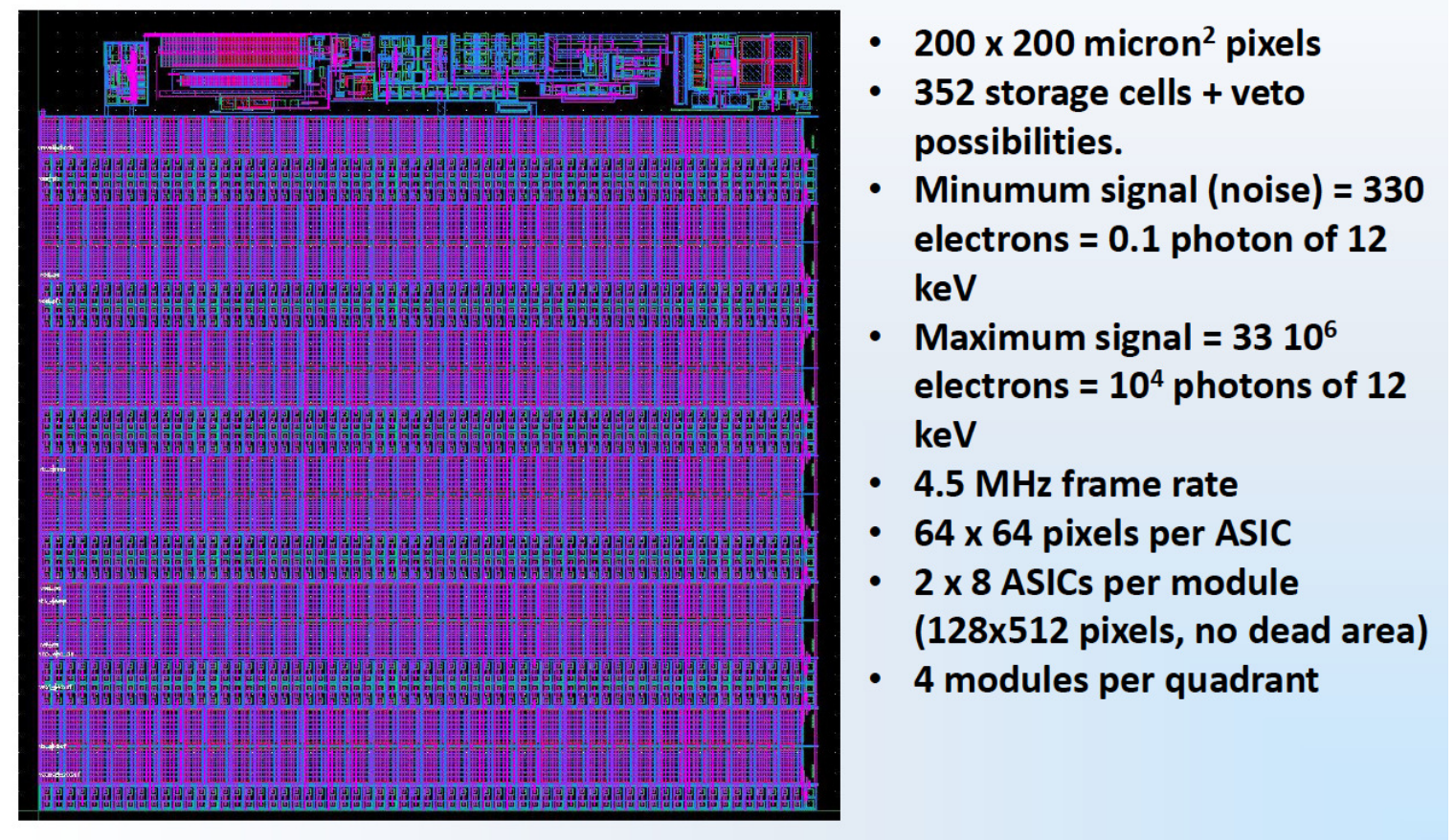

Figure 1: Pixel image and specifications for the AGIPD 1.0 detector: Adaptive Gain Integrating Pixel Detector being developed for EXFEL.

An example of a consensus priority direction is what might be called "The Universal Gated Detector." All imaging systems have many parts (see Figure 2). Historically, projects have optimized all parts for their application; this may not leave enough resources for transformative development in any one part. 


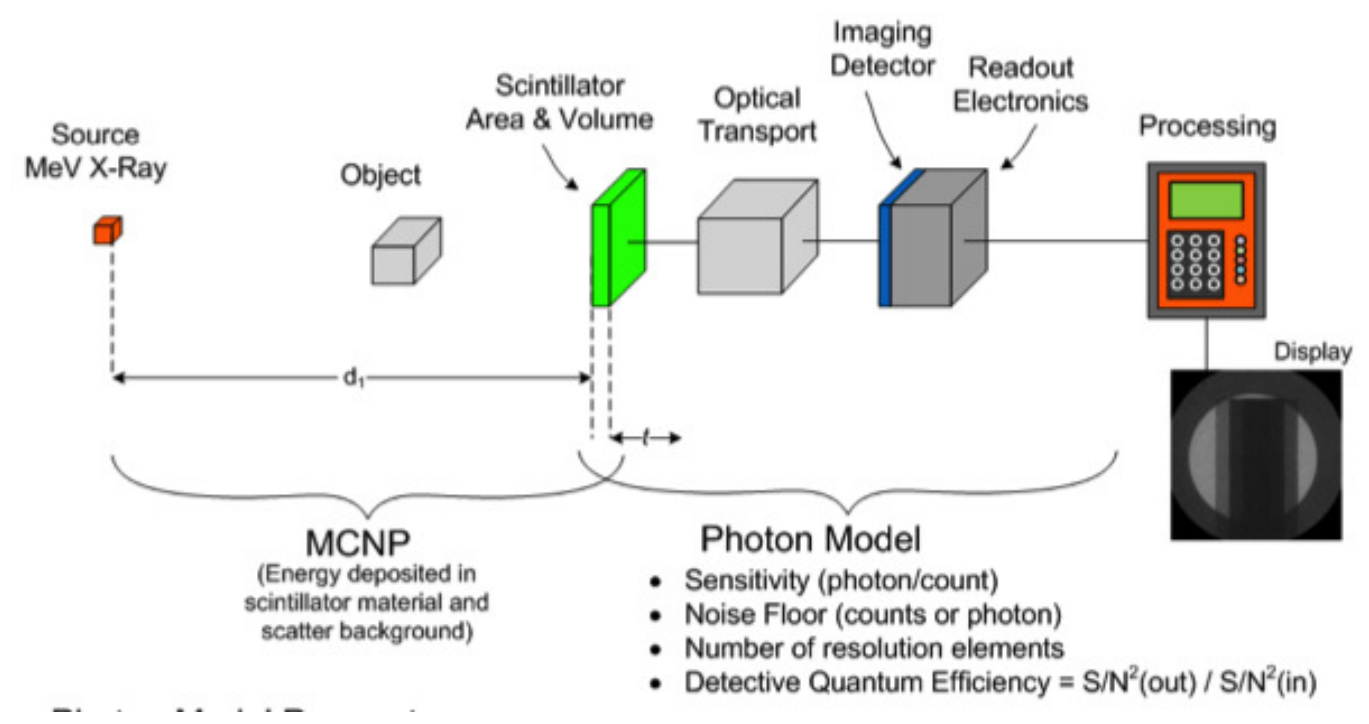

Figure 2: Example of the many parts of an imaging system, each of which can be optimized for an application.

A broad number of applications (light or charged particle sources such as APS, pRad, DARHT for thinner objects, and others) share the need for multi-framing detectors with a common set of requirements; this is a priority research direction set by the "Fast Applications" breakout. And the "Penetrating Radiography" breakout wants these detectors - albeit with a very low noise floor (10 $\left.\mathrm{e}^{-}\right)$for a CMOS detector and may be willing to trade off dynamic range to get that. And the "Ultrafast Applications" breakout wants these detectors - albeit they will trade-off lowerresolution $(1 \mathrm{kX} 1 \mathrm{k}$ rather than $2 \mathrm{kx} 2 \mathrm{k}$ ) to get faster framing (down to $100 \mathrm{psec}$ instead of several nanoseconds). The need for long-term, community-driven and supported, investment in the Universal Gated Detector technology and appropriate variants is clearly called for.

Figure 2 also points out the likelihood of common development needs in other parts of the radiographic chain. Common interest in exploiting the post-9/11 development of new materials and techniques should reinvigorate research into scintillator materials, needed for harder x-ray detection (above 15-20 keV, where silicon begins to get too transparent). The field of materials science is undergoing a transformation from mere observation and discovery to prediction and control of material properties; thus, there is a significant opportunity to take advantage and learn to design required properties of materials such as scintillators. Also, in radiation-hard environments the active electronics often must be shielded and out of the line-of-sight and optimization of the optical coupling system may yield the most significant gains.

The workshop consciously did not tie requirements explicitly to particular experiments, milestones, or even facilities. Rather, more general and broad challenges and needs were considered. All the participants of course brought personal knowledge and experience of particular facilities, and this appropriately flavored the discussions effectively. Initial plenary talks thus set general challenges, 
needs, and requirements for imaging systems relevant to NNSA; some of these are summarized next.

\section{Ultrafast Applications:}

- Operation in harsh EM and neutron environment

- Gated instruments with no spatial or temporal droop

- Gated instruments with less cross talk between the channels

- Visible imagers with less sensitivity to neutron flux

- Large $(6 \times 8 \mathrm{~cm})$ format detectors

Neutron Imaging Applications:

- Multiple frame imaging for neutron imaging applications at NIF

- $\quad 10$ ns exposure times

- Inter-frame times of $10 \mathrm{~ns}$

- 4-10 frames

- $\quad$ Single line of sight.

\section{Penetrating Radiography Applications:}

- Quantum limited

- $\mathrm{DQE}>40 \%$ for $5 \mathrm{mR}-10 \mathrm{mR}$ exposures

- Linearity better than $1 \%$ for 12 bits

- High Resolution $-200 \mu \mathrm{m}$ at object

- $\mathrm{MTF}-0.4 \mathrm{lp} / \mathrm{mm}(50 \% \mathrm{MTF})$

- Large Format

- Proton radiography frame rate $\sim 5 \mathrm{~ns}$ ( $\mathrm{mm} /$ microsecond), exposure time $<1 \mathrm{~ns}$. Electron radiography frame rate $1 \mathrm{~ns}$, exposures $<0.1 \mathrm{~ns}$.

- 50-100 frames desired

Some sources can have many pulses, allowing access to a broad range of time durations. Figure 3 illustrates one particular example from the LANSCE accelerator. The proper imaging systems can support science spanning quasi-static to highly dynamic regimes. The upper bound of possible science can be expanded by improved cameras. 


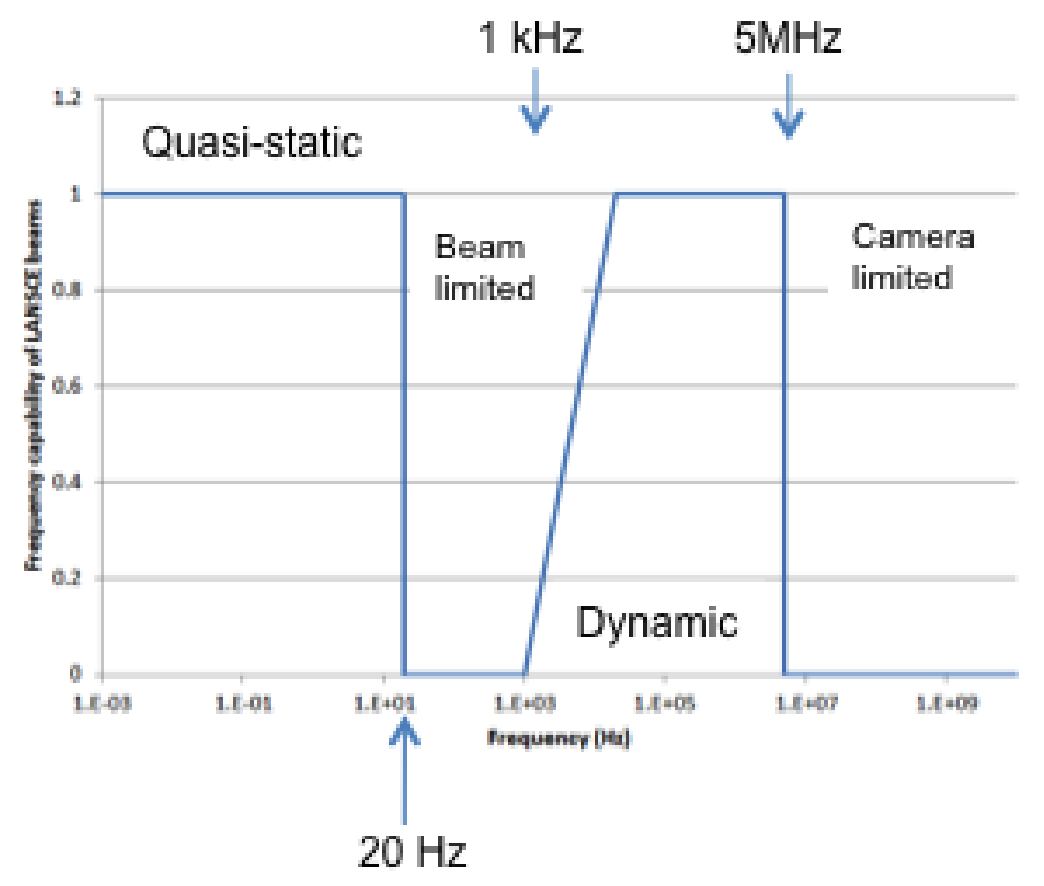

Figure 3: Frequency range accessible to experiments on the LANSCE proton linac such as pRad. The macropulse repetition of the linac creates the hole between $20 \mathrm{~Hz}$ and $1 \mathrm{kHz}$; science beyond $5 \mathrm{MHz}$ is possible with improvements in camera technology.

\section{Fast Applications:}

- Both single event (such as shock driven) and non-single event but timeresolved science applications exist.

- An area detector can record multiple events on a single detector frame to generate statistics. Counting pixel-array area detectors (PILATUS) are ideal for these non-single event experiments.

- Single-event experiments require the use of an area detector with multiple framing capability. In synchrotron-based experiments the time between frames (the inverse frame rate) depends on the storage ring fill pattern. For a synchrotron like APS this presently ranges from 11.4 ns to 153 ns to as long as $1.59 \mu \mathrm{s}$.

- Extremely high instantaneous count rates per detector pixel $\left(10^{14} \mathrm{~Hz}\right.$ possible).

- To maximize light throughput, usually "pink" beams with a spread in energy are used. Typical design points for x-ray energies are hard (several-15 keV) to very hard (15 keV-50 keV).

A common theme that emerged from the workshop was the opportunity to capitalize on developments of the past decade in new materials. Post-9/11 investment by national and homeland security sponsors, investment in national nanocenters by the Office of Science, and the major emergent digital imaging 
markets have driven a wide variety of major improvements in materials useful in imaging.

One example is the growth in the number of available scintillator materials with excellent properties, including the ability to, with research, "tune" the properties by controlling dopant levels. These include both materials able to be grown in "columnar format" which provides transverse collimating of the scintillator light (an excellent example being $\mathrm{Lu}_{2} \mathrm{I}_{3}$ :Ce with a density of $5.6 \mathrm{~g} / \mathrm{cm}^{3}$, an effective $\mathrm{Z}$ of 59.7 , a light yield of 115,000 photons/MeV with peak emission wavelength of $540 \mathrm{~nm}$ and a primary decay time of $28 \mathrm{~ns}$ ) and materials of the inorganic "garnet" family that can be pressed into large format (an excellent example being GLuGAG:Ce with a density up to $7.1 \mathrm{~g} / \mathrm{cm}^{3}$, a light yield of $\sim 60,000$ photons $/ \mathrm{MeV}$ with peak emission wavelength of $540 \mathrm{~nm}$ and a primary decay time of $50 \mathrm{~ns}$ ).

\section{Priority Research Directions}

\section{Ultrafast Applications}

\section{Introduction}

This section is concerned with the detection of $x$ rays between 2 and $100 \mathrm{keV}$ with gating times of less than 200 ps and with significant spatial resolutions that exceed $100 \times 100$ pixels over the field of view. A trade-off between resolution and high speed gating was considered necessary for practical detector construction. The detection area of ultrahigh speed x-ray imaging, considered here, shares challenges and opportunities in radiography, dynamic diffraction, lens-less imaging, ICF implosions and a slew of other HED applications. Ultrafast applications share many of the challenges, limitations, and concerns identified in the other areas in this workshop, such as detection of high-energy x rays with high efficiency, the need for fast scintillators, and read out of the signals in harsh environment. We identified several areas of research that would help in addressing the identified major issues. First, is the conversion of the radiation to electrons. Efficient, fast photocathodes are required for x-ray energies above $10 \mathrm{keV}$; an effort similar in size and scope to the work undertaken by Henke and later collaborators on low-energy $(<10-\mathrm{keV})$ photocathode characterization and development. A related requirement is the improvement of MCP design and construction, particularly in photocathode deposition technology. Second, is radiation-to-optical conversion where the optical properties of the material are changed sufficiently, due to the interaction of the radiation and sensor medium, that optical interrogation of the sensor and conventional ultra-fast optical processing techniques may be applicable. Third, finding fast readout storage media allowing for increased number of images to be recorded within a given short period of time. Fourth, application specific materials $R \& D$ including development of fast scintillating material that has good conversion from $x$-rays to visible light and very fast $<<1$ nanosecond decay times, and the development of materials where the index of refraction would change sufficiently to affect the interrogation of the material with visible light, admitting the use of well developed ultra-fast optical recording techniques. For example, GaN semiconductor materials have sub-picosecond relaxation time, and exceptional radiation hardness. 
Implementing these research directions would allow us to address high-leverage enabling technologies for ultra-fast recording that will provide experimental access to a larger parameter space than is currently available. This includes measuring properties of larger and/or denser objects, improved DQE for the detector helping improve the quality of imaging, and measuring faster processes with the shorter gate times. A collateral benefit is that these developments will have broad scientific impact, benefitting many fields of research that require advanced, high-speed, imaging technology.

\section{Gated x-ray detection at 20-60 keV}

There are a number of experiments that require multiple framed images to be acquired at x-ray energies above $20 \mathrm{keV}$. The requirements are driven by a desire to perform "fast" radiography of "thick" objects and a variety of dynamic materials studies, i.e. materials strength and equation of state at high strain rates.

The required imaging systems must provide 20-40 frame Mega pixel images, with an exposure time of $\sim 100 \mathrm{ps}$, a frame interval varying between 0.3 and $4 \mathrm{~ns}$, and an extinction ratio of 1000:1 with a spatial resolution of better than $100 \mu \mathrm{m}$ in the object plane. At present there is no "good" technical solution to this metrology requirement. Direct detection is challenging due to constraints imposed by the conflicting requirements of high detection quantum efficiency and high speed. Technical innovation is needed.

Promising approaches include conversion of the radiation signature to enable utilization of conventional optical imagers (CCD cameras) or electron imagers (CMOS/HPAD cameras). Advanced electron detection concepts require additional research and development to producing high efficiency photo-cathode materials and the development of ultrashort-time recovery micro-channel plate amplifiers. Optical conversion at $\sim 100$ ps requires research investment to develop new fast scintillator materials which exceed the state of the art decay time and photon yield performance by more than an order of magnitude. Niche applications for advanced radiation-to-optical converter concepts are currently in the research phase, but more broadly defined goals are necessary to expand the applicability of these developments.

\section{Gated x-ray detectors at 2-20 keV}

Gated x-ray detectors over the energy range spanning 2-20 keV are needed for a variety of imaging applications including dynamic material studies at high strain rate, particularly imaging of dynamic diffraction patterns of shocked materials, measurement of laser entrance hole closure in indirect drive ICF experiments and high temporal resolution spectroscopic imaging of imploding cores (self-emission) in ICF implosions.

Here, the required advancements include the development of electromagnetic pulse resistant, multi-frame imagers with frame acquisition times of less than $100 \mathrm{ps}$ capable of recording 20 frames at a 100-ps inter frame time. A resolution between 
1000x1000 for dynamic diffraction and $\sim 4000 \times 4000$ pixels for spectroscopic imaging, and a 1000:1 dynamic range would be necessary.

For x-ray energies below $10 \mathrm{keV}$, direct detection with silicon photo-sensors is highly effective, as are electro-optical conversion systems, photocathode, microchannel plate amplifier and phosphor conversion systems already in use.

Direct detection is not practical for exposure times of less than $50 \mathrm{ps}$, due to the limitations of charge transport; below $\sim 5 \mathrm{ps}$, strip-line gated MCP detectors are no longer practical due to the limitations of gate propagation. At $\sim 1$ ps frame time one of the research-phase ultrafast technologies will probably be required.

For x-ray energies above $10 \mathrm{keV}$, the efficacy of direct detection is questionable therefore additional effort will be required to develop optimized converter technologies including hard x-ray wavelength up-conversion to visible and infrared photons, novel photocathode materials and improved micro-channel-plate architectures.

\section{Ultrafast few-picosecond imaging}

Current framing cameras rely on gating an image using an induced gain across an MCP with a short (few 10s of picoseconds) travelling electrical pulse. The gain of the MCP depends on voltage to the $9^{\text {th }}$ power, reducing the effective gating time to as short as 30 ps at any position of the MCP. However, different positions across the detector have their gate at different times dependent on the propagation speed of the electrical pulse.

Simultaneous gating of the 5-20-keV x-ray image across the detection plane, 0-10ps gating time with, if possible, adjustable 10-30 ps interframe time, and greater than 6 to 8 frames per capture is required for this application. This is a tough challenge for electrical based systems, which may require development of multiple simultaneously but independently gated small areas across the detection plane while insuring that the electron transport of the amplified electron signal maintains the required spatial and temporal resolution. In recognition of the difficulty, an approach could be to accept 100x100 pixels of effective spatial resolution with an extinction ratio of about 1000:1. Furthermore, since by necessity the observed events occur on a sub-nanosecond time scale from small objects that may also emit significant neutrons and high frequency EMP radiation, proper EMP and neutron insensitivity or hardening would be necessary.

Alternative optical techniques, initially showing promising results, need to be extended. These have the advantages of remote readout, hence insensitivity to neutron and EMP radiation, and the use of well characterized and well developed optical recording techniques. But it also requires that the optical setup maintains the temporal resolution and simultaneity across the detection plane, a task that may be eased by the use of computer controlled spatial light modulator to adjust the delay across the optical wave front. 
The creation of such a camera would have immediate impact. Examples include imaging emitted $x$ rays to measure the spatial distribution of reaction history energy heating in the core of imploding ICF capsules, the spatial distribution of fluorescent or emitted radiation from targets excited by the LCLS x-ray beam, measuring ultrafast processes of electron-ion coupling in strongly coupled plasmas, and other fast processes or events occurring in matter at extreme conditions.

\section{Novel materials and techniques}

Many of the approaches to ultra-fast x-ray detection rely on conversion of the x-ray signal to the optical domain. In these cases, it may be possible to utilize a "general purpose" camera design that is useful for a variety of imaging applications. There is very high leverage in adopting a development strategy that strives for an optimized "general purpose" camera architecture and simultaneously invests in performing fundamental materials $R \& D$ to develop application-specific scintillator materials.

Creating a fast scintillating material that has good conversion from $\mathrm{x}$ rays to visible light, and very fast $\ll 1$ nanosecond decay times, would allow the use of welldeveloped optical recording techniques. Complementary materials $R \& D$ is required to develop fast refractive index change sensors that admit interrogation of the sensor using visible light and allow the use of the ultrafast optical techniques. There are several candidate material systems worthy of investigation, and tighter coupling between the NNSA x-ray imaging and materials science community is a prerequisite to defining promising areas for future focused $R \& D$.

Most of the existing x-ray "imaging" systems rely either on point projection or pinhole camera implementations. To increase the signal and/or select the wavelength, added emphasis should be applied to the development of monochromatic, finite-conjugate, high-x-ray-energy imaging systems.

\section{Fast Applications}

\section{Motivation}

Detector requirements for existing and new facilities coming on-line in the near future have been the subject of much interest in the x-ray diffraction and imaging (both x-rays and protons) communities. Techniques such as $\mathrm{x}$-ray diffraction, phase contrast imaging, and radiography, when coupled with time resolved measurements in the nanosecond to microsecond time domain, require detectors with properties that are not currently available commercially. Detectors that are capable of recording very high instantaneous photon flux over short time durations (ps to ns), with multiple framing capabilities are feasible and in some cases have been demonstrated (the adaptive gain integrating pixel detector developed for use at the European XFEL is one example); however, specific properties and specifications need to be adapted for the applications discussed in this planning meeting. Current imaging technology is limited to relatively slow frame rates and small numbers of frames. A variety of experimental programs currently in progress, utilizing $\mathrm{x}$ rays and protons as probes, would benefit from the improved temporal and spatial resolution that will be enabled with more advanced detector technologies. Two 
areas where significant improvements can be made are framing cameras for $\mathrm{x}$ rays and visible light and advanced scintillators that are faster and brighter than currently available options. An advanced framing camera for direct x-ray detection would allow more frames to be recorded at faster frame rates and with lower noise than currently possible. Scintillators are expected to play an important role in the detection of high energy $x$ rays and protons where imaging spatial resolution on the order of one micrometer is desired. By detecting the scintillator light output with a fast framing visible light camera as opposed to the current practice of using gated intensified CCDs it should be possible to obtain more frames with lower noise. A description of a hybrid pixel array detector based camera, desired scintillator properties, and a path forward are outlined below.

\section{Fast Framing Camera}

The array of capabilities and science needs across the NNSA facilities are individually unique, but many applications possess a basic set of detector needs. The need for a high frame rate camera for visible light and/or $\mathrm{x}$ rays cross-cuts many of the applications at NNSA sites. These baseline capabilities applicable to many of the fast (ns) applications are identified in Table 1 below. We anticipate that the baseline capability will meet $\sim 80 \%$ of the needs of the community and will be directly pertinent to DCS at APS, proton radiography at LANL, the Z facility at SNL, and others. Additional specific modifications may meet unique needs of individual facilities.

Table 1: Baseline Capabilities

\begin{tabular}{ll}
\hline Frames & $10+$ \\
Inter-frame time & $1-500 \mathrm{~ns}$ (tunable) \\
Pixel Size & $25 \mu \mathrm{m}$ (imaging) \\
& $150 \mu \mathrm{m}$ (diffraction) \\
& $1024 \times 1024$ \\
Number of Pixels & (minimum) \\
Well Capacity & $3,000,000$ e- \\
& Visible $-12 \mathrm{keV}$ \\
Sensitivity & $12-35 \mathrm{keV}$
\end{tabular}

One possibility for achieving a camera that possesses these baseline capabilities is based on hybrid pixel array detector (HPAD) technology. An HPAD consists of a semiconductor diode wafer as a sensor and a CMOS based wafer containing an application specific integrated circuit (ASIC) for processing and storing the charge generated in the sensor. There are active HPAD camera projects in development. These cameras show great promise and are close to the desired characteristics, but more work is necessary to improve the performance, size, integration time and inter-frame rate.

Integrating HPADs are being developed to accommodate high-flux applications such as those at free electron laser sources and third generation synchrotrons. Unlike 
photon-counting pixel array detectors (e.g. the PILATUS) which are commercially available and are routinely used on low-flux lab-based sources, the likelihood of integrating HPADs becoming an "off the shelf" item is small due their limited (but extremely important) applicability. It is crucial that NNSA laboratories are involved in their development, with the work either in house or in partnership with industry so that mission-relevant capability is ensured. Cameras from such projects need be made available to multiple NNSA facilities for testing and use. An example of such a HPAD detector project is shown in Figure 4 which displays an image of a prototype HPAD detector developed at Sandia which can record four frames.

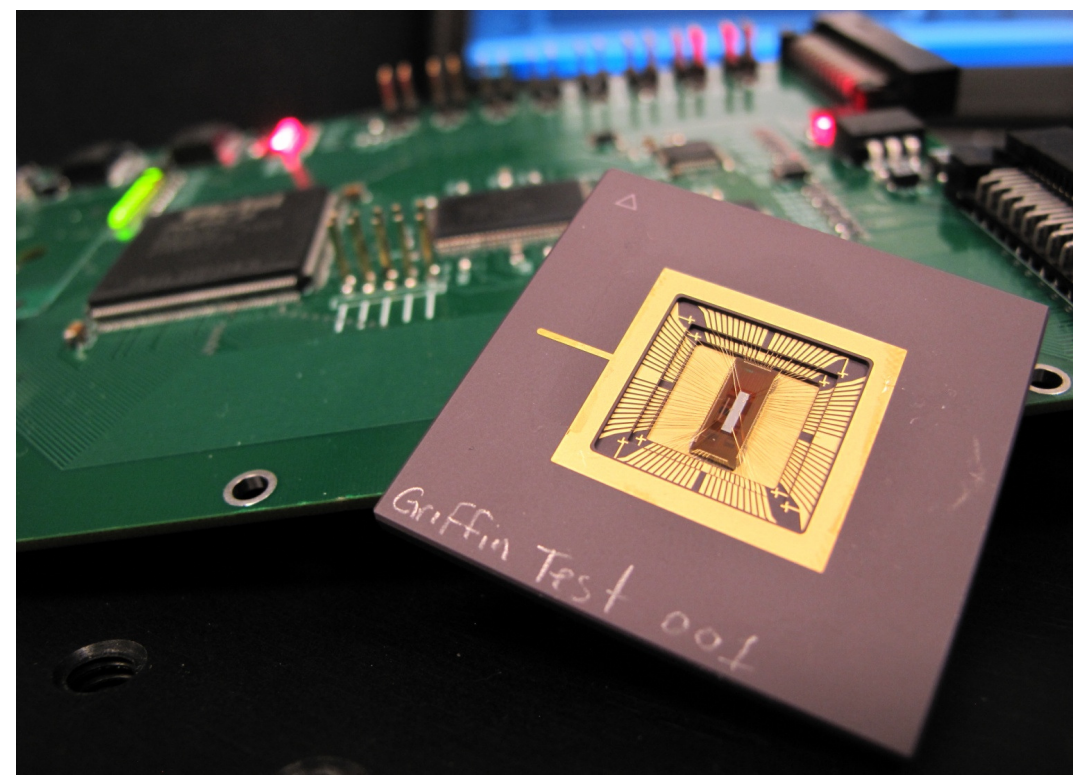

Figure 4: Photograph of a SNL 128x15 pixel prototype hybrid pixel array detector.

This prototype detector utilizes a $25 \mu \mathrm{m}$ sensor thickness to achieve inter frame times as short as $1 \mathrm{~ns}$ (see Figure 5). However, the $25 \mu \mathrm{m}$ silicon sensor thickness limits the quantum detection efficiency for hard $x$ rays $(>5 \mathrm{keV})$; the quantum detection efficiency for hard $\mathrm{x}$ rays can be improved by increasing the sensor thickness, but with a corresponding reduction in frame rate due to increased charge collection time.

Two phases of detector development based on existing technology developed within the NNSA complex are recommended to fully utilize NNSA's unique facilities. The first phase is expected to last approximately 3 years and should produce a detector that will immediately be useful for measurements at NNSA facilities. The second phase of the project is expected to require several additional years of development and would extend the detector's capabilities making it directly sensitive to hardenergy x rays (15-35 keV), utilize large active areas (Megapixel), and employ advanced semiconductor manufacturing processes. 


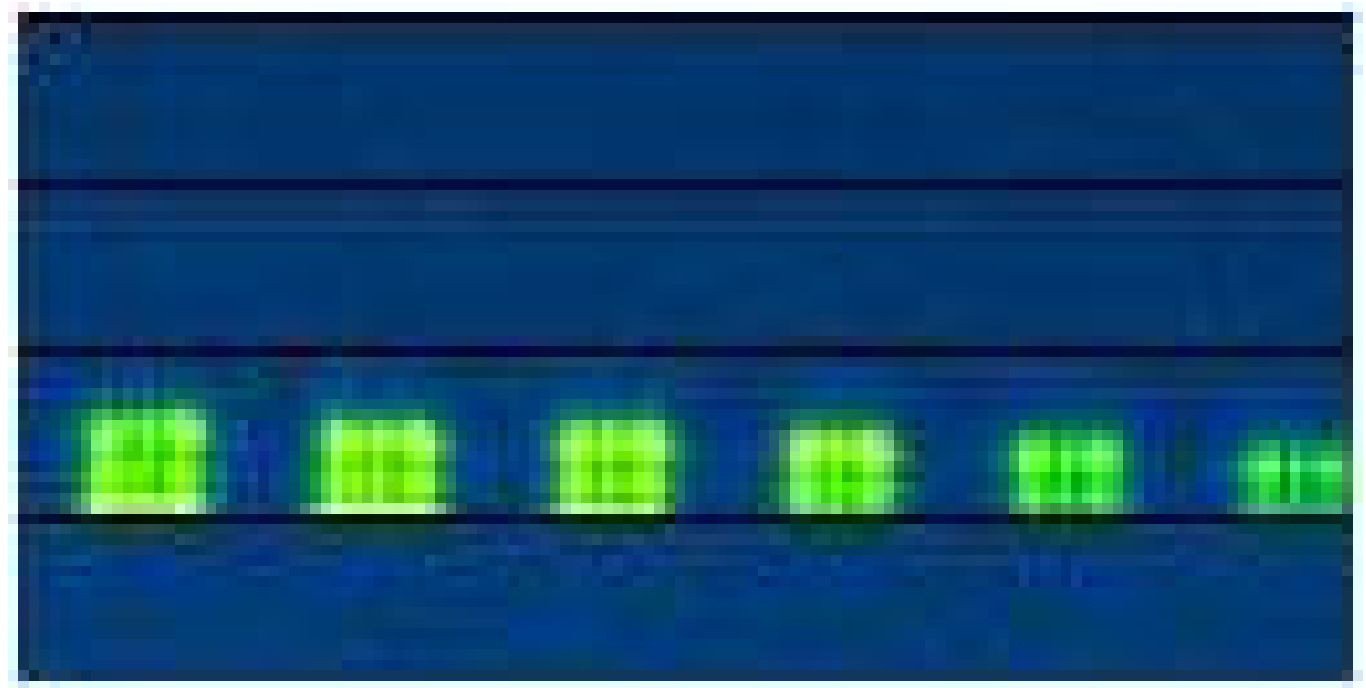

frame 1

frame 2

frame 3

frame 4

Figure 5: Images acquired with SNL prototype camera when illuminated with pulsed optical laser masked with a mesh screen to provide spatial structure. Frame integration time set to 1 nsec and time between frames set to 1 nsec.

In Phase 1, a detector with the ability to record 10 frames sequentially with a minimum inter frame time of less than $10 \mathrm{~ns}$ is desirable. The properties of the Phase 1 detector should be determined through a combination of opinions and sitespecific requirements from the NNSA facilities. With these basic characteristics defined, chip development along with integration into a deliverable camera system would proceed side by side. Back-end development and packaging for reading out the data and communicating with the detector could be provided by NSTec or industry partners. A set of cameras could be produced in the 3-year time frame, distributed to the stakeholders and tested at each site. It is anticipated that feedback from the individual users will lead to a more robust and application-specific second generation camera.

Phase 2 is a longer time frame project (several years) requiring more significant advances in detector technology. The breakout session identified several specific areas that need to be pursued in order to fully utilize current and future experimental resources. Two areas in particular, if successful, would provide significant advances in capabilities: direct detection of hard $\mathrm{x}$ rays and 3D stacking technology. Funding should be directed towards incorporating high-Z semiconductor materials such as Ge or GaAs as the sensor element in HPADs; a high$\mathrm{Z}$ semiconductor can be made thin which allows for short inter-frame times ( $<10 \mathrm{~ns}$ ) while still having a reasonably high quantum detection efficiency for $\mathrm{x}$-ray energies up to $30 \mathrm{keV}$. In addition to direct detection, 3D stacking technology promises to greatly increase the speed of data acquisition, and allow for a reduction of pixel size since the area required for data processing electronics is greatly reduced on the surface of the chip. 


\section{Scintillator Improvements}

For x-ray energies greater than about $15 \mathrm{keV}$, silicon based sensors of reasonable thickness become transparent which reduces the quantum detection efficiency. Direct detection using high-Z semiconductors is an aspect of the path forward, but with a long-term time horizon. Scintillators offer a possibility for hard x-ray imaging in the near term while maintaining high resolution imaging capability. High frame rates desired for most of NNSA's experimental programs necessitate fast $(<20 \mathrm{~ns})$ decay times, high $\mathrm{x}$ ray to visible conversion efficiency, and low afterglow.

Table 2: Desired Scintillator Properties

$\begin{array}{ll}\text { Decay Time } & <20 \mathrm{~ns} \\ \text { Afterglow } & \text { None } \\ \text { Light-Output } & 50,000+/ \mathrm{MeV} \\ \text { Wavelength } & 450-600 \mathrm{~nm} \\ \text { Z-effective } & 50+\end{array}$

Research has gone into developing and optimizing new scintillator materials in recent years, and several promising materials (e.g. $\mathrm{LuI}_{3}: \mathrm{Ce}, \mathrm{LaBr}_{3}: \mathrm{Ce}, \mathrm{LYSO}, \mathrm{BaF}_{2}$ ) that exhibit high visible light output and fast decay times have been discovered. Rigorous testing of new scintillator materials is required to characterize the decay times, brightness and afterglow properties. Additionally, it has been demonstrated that by varying the growth process properties such as decay time, brightness, optical transparency, and resolution can be fine-tuned. Further effort towards developing new scintillator materials, studying novel structured scintillators, and optimizing their properties for specific applications should result in significantly improved imaging capabilities.

\section{Penetrating Radiography Applications}

\section{High Sensitivity, High Resolution, Low Noise Imaging Systems}

Penetrating radiography applications tend to be light-starved imaging applications due to the combination of x-ray/object penetration, radiation-to-light conversion, and image optical transport used in hard x-ray environments. Imaging systems (see Figure 6 for example) use scintillators for radiation-to-visible light conversion with an optical relay to image the scintillator onto a remote recording camera. We address pulsed and high resolution applications in the NNSA complex, and much interest is placed on multi-image dynamic experiments. Current configurations utilize single-pulse and multi-pulse $\mathrm{x}$-ray machines along with accelerator facilities for proton radiography. 


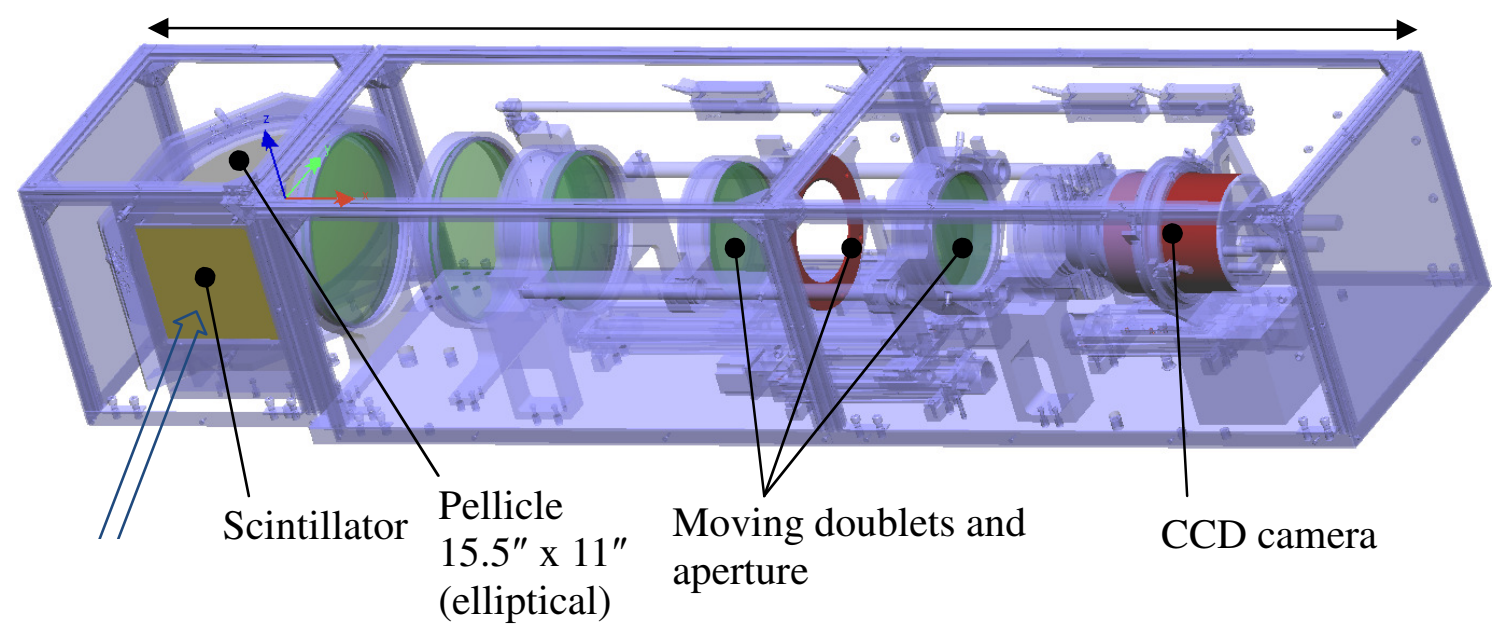

Figure 6: Scintillator with zoom lens and CCD camera system.

Research and development areas focus on improving signal-to-noise (SNR) characteristics of radiographic imaging systems. Signal-to-noise characteristics are often reported as detective quantum efficiency $\left[\mathrm{DQE}=(\mathrm{S} / \mathrm{N}){ }^{2}{ }_{\text {out }} /(\mathrm{S} / \mathrm{N})^{2}{ }_{\text {in }}\right]$. Image signal-to-noise can be improved with better radiation conversion in the scintillator, direct energy detection, and/or better optical camera detection (see Figure 7). Single-image radiography applications may utilize non-gated, staring camera, with relatively long exposures which can incorporate astronomy-style imagers. This type of camera would benefit from higher-gain electronics with $>2$ electrons per count signal conversion along with low read-out noise, $1 / 2$ electron or less. Larger pixels, $\sim 50$ microns, could be used to increase signal collected per pixel. This area needs attention in the near term of the next few years, and may require collaborative development with industry. For the purposes of the workshop this development was considered to provide incremental improvement to existing capabilities. The primary research directions (PRDs) identified below may require longer term development and are expected to provide broader impact to the program.

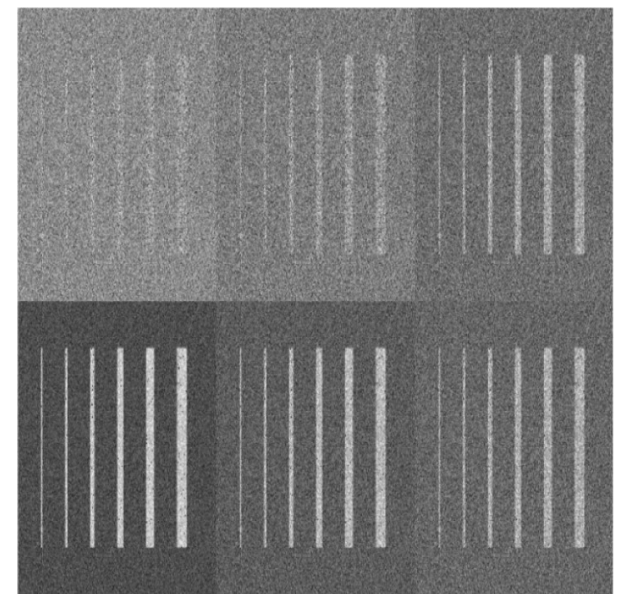

Figure 7: Image signal-to-noise samples illustrating DQE from $0.5 \%$ to $100 \%$. 
Three PRDs are discussed:

- Gated framing imagers

- Scintillator development

- Direct energy detection imagers

\section{Gated framing imagers}

The radiographic facilities in the NNSA complex include a wide range of capabilities. It is a challenge to attempt to design an imaging system that would be equally applicable to every source; however, there are several elements of such a system that can be generally applied. One of these is gated imaging. Most facilities are pulsed x-ray or proton sources, some single pulse and others are multi-pulsed to provide snap-shot radiographs of a dynamic experiment. In most cases x-ray backgrounds are reduced when the detector collects data only during the relevant flash.

\section{Challenge and Opportunities}

In some experimental configurations or tests the detector is "light-starved" or in other words has a signal-to-noise ratio approaching 1. In such light-starved scenarios the image noise levels can have large components due to detector dark current or camera read noise. Additional contributions come from other radiation sources or pulses whose contributions would ideally be temporally discriminated. Most applications also require large fields of view and high spatial resolution (large pixel count).

\section{Research Direction}

Several avenues exist to gate an image. Micro-channel plates intensify the number of photons actually reaching the detector array as well as effectively gating the light down to $1 \mathrm{~ns}$ in duration. This could be useful in situations where one desires to amplify the light well above the read noise of the detector. However in the "shot noise" regime, this may actually degrade the SNR as well as resolution by introducing image blur.

On-chip gating is likely a better alternative as long as the noise level remains small. The standard detector array options, CCD or CMOS, have trade-offs in this regard. It is much easier to gate a CMOS, but the read noise tends to be much higher. Maximizing quantum efficiency in the relevant wavelength band is also important.

A back-illuminated silicon pixelated array detector that is bump-bonded to a gated CMOS readout integrated chip (ROIC) may produce advanced multi-frame hybrid imagers and focal plane arrays (FPA). Early efforts in this technology have shown success in providing gated multi-frame exposures; however, detector noise and array dimensions need improvement. 


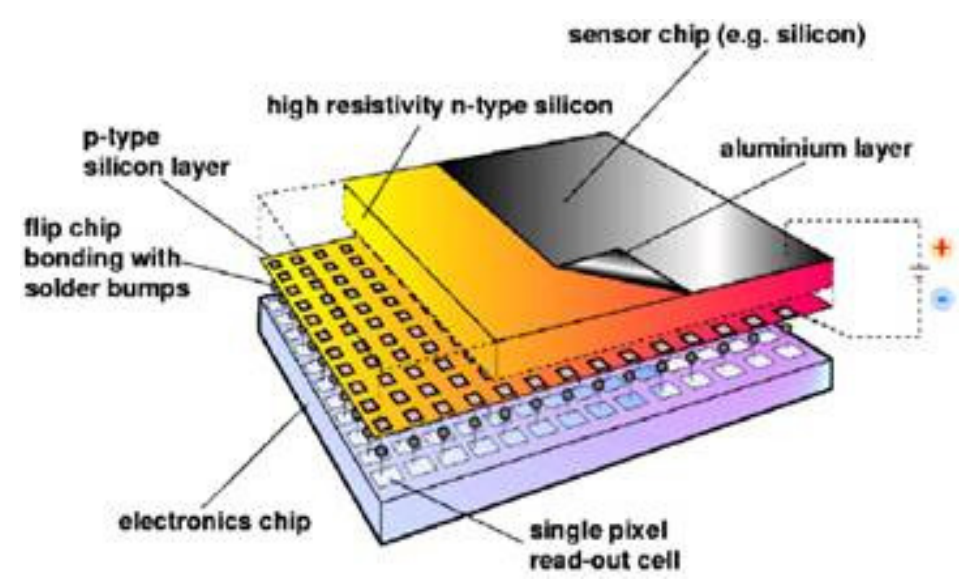

Courtesy DESY Photon-Science Detector Group, Heinz Graafsma

Figure 8: Hybrid image sensor layers

\section{Requirements}

High spatial resolution over a large object field of view requires a large number of resolution elements. Spatial sampling in the range of 2000 elements has shown high quality results with staring array cameras. We look to achieve this type of resolution in a gated imager. The quantum efficiency $(\mathrm{QE})$ of back-thinned silicon detector arrays achieving $90 \%$ QE for visible light collection from 400 to $600 \mathrm{~nm}$ is required for efficient detection of fast scintillators used in this application.

There is significant trade space between associated ROIC design features versus signal quality. Multi-pulse imagers may accept fewer resolution elements or somewhat higher noise levels for the advantage of recording several frames of data. In the area of detecting the best scene contrast and image detail a gated single frame imager is preferred if that allows higher pixel count and/or better noise characteristics.

The desired characteristics are summarized below:

- $2 \mathrm{k}$ x $2 \mathrm{k}$ imager array

- High QE detection, $>=90 \%$ sensor QE

- Best single exposure

$\circ$ Target read noise $<1 \mathrm{e}-$

$\circ$ gate $\sim 200 \mathrm{~ns}$

- Multi-frame imager - 8 to 10 Frames

$\circ$ read noise $<10 \mathrm{e}-$

- Frame exposure $50 \mathrm{~ns}$

- Frame rate $5 \mathrm{MHz}, 200 \mathrm{~ns}$ period

○ Dynamic range 1,000:1 for 12-bit recording

\section{Advantages and Impact}

Gated imagers can provide a great reduction in x-ray scatter background associated with radiographic imaging. Much effort goes into shielding and collimation to 
reduce scatter but often the object itself creates substantial scatter. Scattered x rays can raise the background level of the scintillator limiting the lower threshold of detectable penetration. Scatter is also associated with induced pixel staring in the imaging detector.

Some radiographic configurations would allow cameras to be gated after the x-ray machine fires to record the image from a bright slower-decay scintillator. Multiframe imagers will support multi-pulse machines and provide more data per dynamic experiment.

- Multi-pulse radiography

- More data points per dynamic experiment

- Resolve image detail with lower backgrounds

- Improve scene contrast at low signal levels

- Ability to radiograph through more material

\section{Scintillators}

\section{Challenge and Opportunities}

High energy radiography always (for now anyway) requires a conversion of the $\mathrm{x}$ rays or other particles such as protons into (usually) visible photons which can be then detected using optical camera systems. Scintillators are used for x-ray energies anywhere from $100 \mathrm{keV}$ to near $20 \mathrm{MeV}$. The effectiveness of the scintillator includes the following factors or characteristics which sometimes compete with each other:

- High radiation absorption. This requires dense material or thick scintillators.

- High conversion efficiency - the number of photons per unit energy deposited. This is on the order of 10,000 to 100,000 photons/MeV.

- High resolution. Limit as much as possible the lateral spreading of light and secondary charged particles. This encourages thin monolithic or pixelated thicker scintillators.

- Fast. If utilizing a gated or multi-frame camera system, how fast the scintillator decays determines the minimum gate time and frame rate. Conversely, if multi-source or multiple pulses exist, afterglow may contribute a background which introduces an extra source of noise.

- Low index of refraction to reduce light reflection losses in the lens coupled image.

Traditionally compromises exist when considering stopping power and resolution. A thick scintillator, which stops more of the beam, also tends to spread the light over a broader area. In this regard maximizing the material density helps a great deal. In addition, the experiment or facility usually requires a certain field of view at the detector plane. 


\section{Scintillator Development}

Over the last several years there have been considerable advances in new scintillator materials which offer significant improvements over older types. They are often faster, brighter, and have can have lower intrinsic blur. Development has been chiefly motivated by medical imaging (higher resolution) and the homeland security community (brighter) applications. However, scintillator properties required for radiographic applications are unique, requiring tailored temporal response at large form factor (high spatial resolution).

There is not a one-size-fits-all scintillator. This is especially true with respect to spatial resolution. One strategy to reduce the spreading of light is to produce socalled columnar crystals that create internal "light pipes" that channel the scintillation towards the surface instead of laterally throughout the entire crystal. The spatial scale of these structures is tens of microns. A number of materials can be grown this way. The question remains on whether the thickness, size, and decay time is satisfactory. We would like to investigate how the efficiency changes when the column size becomes smaller and the relative surface area increases. Another technique is to force pixelation on the material that physically blocks the light from traveling from one pixel to the other. This can be done by assembling individual scintillators together with a reflective material inserted between pixels to confine light to the pixel and minimize optical cross-talk between adjacent pixels, a process which tends to be expensive. A recent advance uses a laser to locally modify the refractive index in a bulk scintillator in a grid pattern which again limits the lateral spreading of light. In either case, the pixelation may or may not be fine enough for the local radiographic requirements.

\section{Requirements}

Not every facility or experiment requires or will necessarily benefit from very fast scintillators. When scintillators are used for fast applications, an inter-frame period of 150-200 ns pushes the scintillator decay time to not much more than $20 \mathrm{~ns}$ in order to minimize afterglow backgrounds. Bright scintillators at this time scale appear possible. Scintillator relaxation times on the order of $10 \mathrm{~s}$ of ns may be a lower limit, as at shorter times signal is lost due to competition of alternative scintillator relaxation processes.

Resolution requirements depend on the facility. If resolution is detector-limited or in situations where the field of view is around $200 \mathrm{~mm}$ or less, a columnar crystal may be more appropriate with detector blur on the order of 200 microns or less. Large fields of view of several hundred $\mathrm{mm}$ may tolerate $0.5-\mathrm{mm}$ pixels which can be accomplished with pixelated detectors which may be thicker as well.

\section{Advantages and Impact}

Increasing the light output of a fast scintillator can be just as effective as increasing the dose output of the accelerator, which in turn may allow radiographing thicker objects. If the speed of the scintillator allows more images to be taken during a dynamic experiment, then the amount of information proportionally increases 
which is an important factor to consider in the cost effectiveness of a facility or experiment.

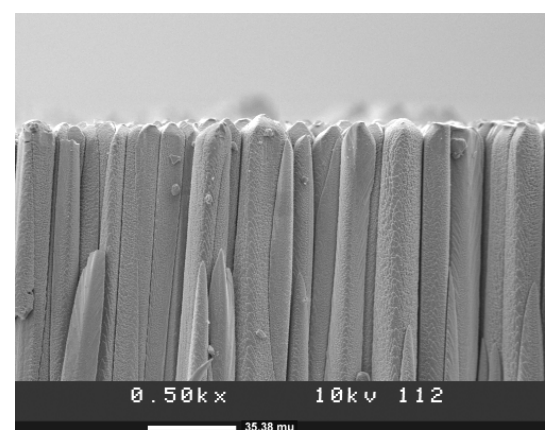

RMD columnar scintillator

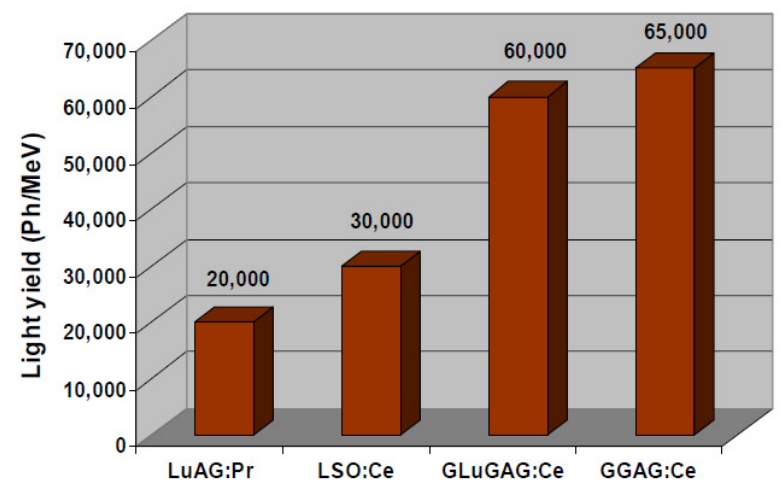

RMD data

Figure 9: Examples of recent advances in scintillator development: (Top) The ability to create thick (multi-millimeter) columnar layers of a variety of high light yield materials. (Bottom) Examples of some of the new ceramic scintillators.

\section{Direct Energy Detection Imagers}

The optical transport, typically an image relay lens or a coherent fiber optic imaging bundle is a key component of many radiographic imaging systems. Optical relays are often used in radiography in energies greater than 10 's of keV to keep the recording camera electronics out of the x-ray beam. The scintillator/optical relay serve a critical role, but they are not very efficient in overall $\mathrm{x}$-ray photon detection efficiency. Direct energy detectors would provide the ability to image $\mathrm{x}$ rays directly and remove the need for scintillator and relay lens.

\section{Challenge and Opportunities}

Typical CCD, CMOS, or hybrid imagers utilize silicon detector pixel arrays providing charge absorption for visible photons. Higher energy $\mathrm{x}$-ray photons pass through and are not absorbed in the silicon detector layer. Denser or higher Z semiconductor pixels can provide stopping power and absorption of $\mathrm{x}$-ray photon energy. Developing high-resolution pixelated arrays of high Z material is a challenge.

\section{Research Direction}

Semiconductor material such as cadmium zinc telluride continues to be developed for radiation detectors. Some work has successful developing imaging pixel arrays. Continued development is needed to develop appropriate stopping power, pixel 
element resolution and the ability to produce large arrays. Readout application specific IC's (ASICs) development is required but may utilize development discussed earlier in the gated imager section.

\section{Requirements}

- Pixel size of 100 to 200 microns.

- Pixel array dimensions approximately 2400 x 2400 elements desirable (1000 $\mathrm{x} 1000$ is useful).

- Semiconductor dense enough to absorb 1.3-MeV x rays, useful range $100 \mathrm{keV}$ to $>1 \mathrm{MeV}$.

- Resistance to radiation damage.

- Gated readout ASIC is required for multi-frame exposure storage.

\section{Advantages and Impact}

- Allow higher frame rate sampling with elimination of scintillator and associated decay time.

- Direct imaging in photon starved scintillator radiography could provide a large gain in $\mathrm{x}$-ray photon detection by removing the radiation to light converter and optical transport from imaging system and allow radiographs through greater areal density.

\section{Cross-cutting Issues}

Two cross-cutting "priority research directions" (actually more like important areas of program investment) were specifically identified:

Annual C3-sponsored information workshop: This week's workshop brought together a diverse group of participants who learned much from each other. We recommend that Campaign 3 sponsor a regular workshop, not for planning, but for information exchange. It would be an open conference bringing together materials scientists, detector developers, and application users making presentations to share current needs, problems, and opportunities. The workshop might have the first day be export controlled, and the second day open. All presentations able to be made public would be posted to a web-site until the next workshop. A period between workshops similar to what works well for JOWOGs (about 18-months) is not too often but often enough to stay abreast of major developments. This workshop would allow the sharing of knowledge and learning across the complex, and help identify transformative approaches to mission need.

Ensure the Infrastructure in place, both people (collaborations) and equipment (testing and characterization): Successful projects always have met and overcome unforeseen problems. It is important to avoid supporting sub-critical development efforts that don't succeed when they hit a problem. An approach is to encourage and support building "right-size" collaborations on important projects. Having multi-site teams ensures necessary expertise is available to address critical problems that arise. It increases the diversity of the team, allowing a wider set of solutions to be considered, knowledge and use of common lessons learned to be shared, and possibly leading to more transformative and important development. 
Central to this broader collaborative approach is recognizing and encouraging relationships with key industrial partners. It improves timeliness of responding to mission need and reduces time to delivery of new systems. Some types of imaging system development may have a strong market and industry can drive the development; other types may require active investment by mission sponsors to push the technology in important but unique ways. It does require complex-wide prioritization and planning; how to achieve this needs to be left to Science Executives' consideration.

The Infrastructure is not just the people but also the fabrication, testing, and characterization capabilities necessary for the development and in particular identification and solution of problems. The development team having access to testing sources with the correct characteristics is vital to project success. This was not discussed in great detail at the workshop; however, a likely concern is access to hard (20-80 keV) short-pulse x-ray sources for development of detectors. These can be generated relatively simply by a modern "table-top" petawatt laser - but those are million dollar investments.

\section{Summary}

The identified priority research directions are listed here:

- Ultrafast

- Gated x-ray detection at 20-60 keV

- Gated x-ray detectors at 2-20 keV

- Ultrafast few-picosecond imaging:

- Novel materials and techniques

- Large $(6 \times 8 \mathrm{~cm})$ format detectors

- Fast

- Fast framing camera

- Desired scintillator properties

- Penetrating Radiography

- Gated single frame imagers

- Multi-frame imagers

- Scintillator development

- Direct energy detection imagers

- Cross-cuts

- "Annual" C3-sponsored information workshop

- Ensure the Infrastructure in place, both people (collaborations) and equipment (testing and characterization)

There is clear combination into a smaller set. First though, an important point: what can be referred to as the "Universal Gated Detector" is perhaps better described as a "Universal Backplane" which can accommodate optimized application specific "detectors". Synergies exist between the various requirements arising from the camera architecture - i.e. the gating widths, well depth, readout speed, frame rate, etc. Generally, however, the sensor (or converter) end of the detector is very 
application specific. Both the back-end (backplane) and front-end (detector) technical innovation is required and should be assigned equal priority.

1. Development of the "Universal Gated Detector," both a "backplane" as well as different optimized application specific "detectors." It would be a multiframe $(\sim 10)$ imager for optical and soft x rays.

a. And further development into low noise-floor $\left(<10 \mathrm{e}^{-}\right)$for photonstarved applications

b. And further development into phosphors that allow use of hard (20$60-\mathrm{keV}$ ) x rays

2. Interactions with materials scientists and industrial partners on controlling the properties of desired scintillators

3. More application specific needs:

a. Ultrafast few-picosecond imaging

b. Gated single frame imagers

c. Direct energy detection (of MeV photons) imagers

4. "Annual" C3-sponsored information workshop

5. Ensure the Infrastructure in place, both people (collaborations) and equipment (testing and characterization) 
Final Agenda

Monday Morning, March 25

8:15 Welcome - Christopher Werner (NSA)

8:30 Workshop Organization - Jon Kapustinsky (LANL)

8:45 Fast Applications and light sources - Tim Graber (WSU)

9:15 Hi resolution low noise radiography - Barry Warthen (LANL) LA-UR$13-22100$

\section{9:45-10:00 Break}

10:00 Ultra-fast applications and HEDP - George Kyrala (LANL)

10:30 European Developments and the XFEL - Heinz Graafsma (DESY)

11:00 Advanced Sensors and Detectors - Marcel Demarteau ( $\underline{\mathrm{ANL}}$ )

11:30 LCLS Detectors and Beyond - Chris Kenney ( $\underline{\text { SLAC) }}$

12:00 Breakouts - Cris Barnes (LANL)

\section{2:15-1:15 Lunch}

1:15-4:30 Breakout sessions (Rosen, A234, D105)

- 1. High Efficiency, Low Noise Radiography - Stuart Baker (NTec), Tim Webb $(\mathrm{SNL})$

- 2. Nano to microsecond imaging - Christopher Seagle ( $\underline{\mathrm{SNL}})$, Tim Graber $\underline{\mathrm{WSU}}$ )

- 3.Sub-nanosecond imaging - Steve Vernon (LLNL), George Kyrala (LANL)

4:30 Reconvene and report on breakouts (Rosen Auditorium)

\section{Tuesday Morning, March 26}

8:15 Advances in Scintillators, Vivek Nagarkar (RMD)

8:30 Cross-cut issues, Cris Barnes

Breakout sessions continue (Rosen, A234, D105)

\section{0:30 Break}

11:00 Final breakout reports and discussion (Rosen Auditorium)

12:30 adjourn general workshop, breakout session leads prepare final report 


\section{Organizing Committee}

Cris W. Barnes, LANL

Stuart Baker, NSTec

Tim Graber, WSU

Steve Goldstein, NSTec

Jon Kapustinsky, LANL

George Kyrala, LANL

Evelyn Mullen, LANL

Bryan Oliver, SNL

Christopher Seagle, SNL

Steve Vernon, LLNL

Tim Webb, SNL

Chris Werner, NNSA

Larry Wiley, LLNL

Stefan Turneaure, WSU/APS

The workshop could only be a success through the professional and expert support of the workshop staff:

Rose Romero, Event Planner

Elena Fernandez, Senior Public Information Specialist

George Dominguez, Visitors and Badging

Celine Apodaca, Foreign National Support 


\begin{tabular}{|c|c|c|}
\hline Name & E-Mail & Institution \\
\hline Stuart A. Baker & bakersa@nv.doe.gov & National Security Technologies \\
\hline Cris Barnes & cbarnes@lanl.gov & Los Alamos National Laboratory \\
\hline Liam D. Claus & Ldclaus@sandia.gov & Sandia National Laboratories \\
\hline Marcel Demarteau & demarteau@anl.gov & Argonne National Laboratory \\
\hline Daniel Dolan & dhdolan@sandia.gov & Sandia National Laboratories \\
\hline Vincent Douence & vdouence@teledyne.com & Teledyne Technologies \\
\hline Darryl W. Droemer & $\overline{\text { droemedw@nv.doe.gov }}$ & National Security Technologies \\
\hline Eric Dutra & dutraec@nv.doe.gov & National Security Technologies \\
\hline Heinz Graafsma & heinz.graafsma@desy.de & Deutsches Elektronen-Synchrotron \\
\hline Timothy Graber & tim.graber@wsu.edu & Washington State University \\
\hline Ed Gooding & egooding@piacton.com & Princeton Instruments \\
\hline Dana Hargrove & hargrove3@llnl.gov & Lawrence Livermore National Laboratory \\
\hline Russell Howe & howera@nv.doe.gov & National Security Technologies \\
\hline Eugene E. Hunt & huntee@nv.doe.gov & National Security Technologies \\
\hline Michael J. Jones & jonesmi@nv.doe.gov & National Security Technologies \\
\hline Mark D. Johnston & mdjohn@sandia.gov & Sandia National Laboratories \\
\hline Jon Kapustinsky & jonk@lanl.gov & Los Alamos National Laboratory \\
\hline Randolph Rex Kay & rrkay@sandia.gov & Sandia National Laboratories \\
\hline Christopher Kenny & kenney@slac.stanford.edu & SLAC National Accelerator Laboratory \\
\hline Alexi Kilmenko & klimenko@lanl.gov & Los Alamos National Laboratory \\
\hline Kris Kwiatkowski & krisk@lanl.gov & Los Alamos National Laboratory \\
\hline George Kyrala & gak@lanl.gov & Los Alamos National Laboratory \\
\hline Amy Lewis & LewisAE@nv.doe.gov & National Security Technologies \\
\hline Brian Maddox & maddox3@llnl.gov & Lawrence Livermore National Laboratory \\
\hline Robert B. Merl & merl@lanl.gov & Los Alamos National Laboratory \\
\hline Frank Merrill & fmerrill@lanl.gov & Los Alamos National Laboratory \\
\hline Steven Mitchell & mitchese@nv.doe.gov & National Security Technologies \\
\hline Evelyn Mullen & emullen@lanl.gov & Los Alamos National Laboratory \\
\hline Vivek V. Nagarkar & vnagarkar@rmdinc.com & Radiation Monitoring Devices, Inc. \\
\hline Bryan Oliver & bvolive@sandia.gov & Sandia National Laboratories \\
\hline John L. Porter & jlporte@sandia.gov & Sandia National Laboratories \\
\hline Gideon Robertson & garobe@sandia.gov & Sandia National Laboratories \\
\hline Gregory A. Rochau & garocha@sandia.gov & Sandia National Laboratories \\
\hline Christopher Seagle & ctseagl@sandia.gov & Sandia National Laboratories \\
\hline Ke-Xun (Kevin) Sun & Ke-Xun.Sun@unlv.edu & University of Nevada, Las Vegas \\
\hline Kevin Toerne & ktoerne@specinst.com & Spectral Instruments \\
\hline Stefan Turneaure & stefant@wsu.edu & Washington State University \\
\hline Stephen P. Vernon & vernon1@llnl.gov & Lawrence Livermore National Laboratory \\
\hline Thomas B. Waltman & waltmatb@nv.doe.gov & National Security Technologies \\
\hline Jeff Wang & zwang@lanl.gov & Los Alamos National Laboratory \\
\hline Timothy J. Webb & tjwebb@sandia.gov & Sandia National Laboratories \\
\hline Franz A. Weber & WeberFA@nv.doe.gov & National Security Technologies \\
\hline Christopher Werner & \multicolumn{2}{|c|}{ christopher.werner@nnsa.doe.gov NNSA } \\
\hline Larry G. Wiley & wiley1@llnl.gov & Lawrence Livermore National Laboratory \\
\hline
\end{tabular}

